\title{
Clemastine effects in rat models of a myelination disorder
}

\author{
Christopher A Turski ${ }^{1}$, Gabrielle N Turski ${ }^{2}$, Bingming Chen ${ }^{3}$, Hauhui Wang ${ }^{4}$, Moones Heidari ${ }^{5}$, Lingjun Li ${ }^{3}$, Kevin K Noguchi ${ }^{4}$, \\ Cara Westmark ${ }^{1}$, Ian Duncan ${ }^{5}$ and Chrysanthy Ikonomidou ${ }^{1}$
}

BACKGROUND: Pelizaeus Merzbacher disease (PMD) is a dysmyelinating disorder of the central nervous system caused by impaired differentiation of oligodendrocytes. This study was prompted by findings that antimuscarinic compounds enhance oligodendrocyte differentiation and remyelination in vitro. One of these compounds, clemastine fumarate, is licensed for treatment of allergic conditions. We tested whether clemastine fumarate can promote myelination in two rodent PMD models, the myelin-deficient and the PLP transgenic rat.

METHODS: Pups were treated with daily injections of clemastine (10-30 mg/kg/day) on postnatal days 1-21. Neurologic phenotypes and myelination patterns in the brain, optic nerves, and spinal cords were assessed using histological techniques.

RESULTS: No changes in neurological phenotype or survival were observed even at the highest dose of clemastine. Postmortem staining with Luxol fast blue and myelin basic protein immunohistochemistry revealed no evidence for improved myelination in the CNS of treated rats compared to vehicle-treated littermates. Populations of mature oligodendrocytes were unaffected by the treatment.

CONCLUSION: These results demonstrate lack of therapeutic effect of clemastine in two rat PMD models. Both models have rapid disease progression consistent with the connatal form of the disease. Further studies are necessary to determine whether clemastine bears a therapeutic potential in milder forms of PMD.

$\mathbf{P}$ elizaeus Merzbacher disease (PMD; OMIM \#312080) is a rare dysmyelinating disorder of the central nervous system that is inherited in an X-linked recessive manner. Prevalence is about $1 / 100,000-200,000$. The disease results from duplication of or a mutation in the proteolipid protein (PLP1) gene; proteolipid protein (PLP) is the major CNS myelin protein. The majority of boys with PMD have a duplication of PLP1 (60-65\%) while the remainders have a point mutation of the gene. The clinical disease is heterogeneous, with two major subtypes, classical PMD (type I) and connatal PMD (type II). Affected boys/men have significant neurologic symptoms consisting of ataxia, rotary nystagmus, pyramidal signs, seizures, extrapyramidal movement disorders, psychomotor delay, and intellectual disability. Most remain in life-long chronic care $(1,2)$.

To study PMD, we and others have turned to animal models of the disease (3-7). Duncan and colleagues have studied two of these in detail, the myelin-deficient rat (a point mutant) and a large animal canine model, the shaking pup $(s h p)$ (also a point mutant) (8-12). New observations in the canine model of PMD suggest that there is a gradual spontaneous differentiation of oligodendrocyte progenitor cells (OPCs) in the spinal cord but not brain, which correlates with clinical improvement in those dogs over 2 years of age in which more myelin is seen microscopically in the spinal cord (12). These findings suggest that drugs that could promote differentiation of OPCs earlier in the brain and spinal cord of PMD patients could help advance myelination and result in functionally significant neurologic improvement. Recent exciting new data, from studies of high-throughput screening of drugs that promote the differentiation of OPCs to mature oligodendrocytes (OLs), have identified compounds used in current clinical practice for other indications. Mei et al. (13) reported that a cluster of antimuscarinic compounds enhance oligodendrocyte differentiation and remyelination. One of these compounds is clemastine fumarate, an anti-histamine and anticholinergic medication that is licensed in the United States for treatment of allergic conditions. Clemastine was also shown by Mei et al. (13) to promote in vivo remyelination in the lysolecithin-induced demyelination mouse model.

The significance of the findings (13) for PMD is as follows: There is a lack of mature OLs in PMD in the white matter of the models studied. However, this changes with time, as has now been shown in the shp. As the shp ages, more myelin is seen in the spinal cord and there is evidence that this results from a delayed but still ongoing differentiation of OLs (12). While the spinal cord appears to be almost normally myelinated, the brain lags behind. The hypothesis therefore is that the promotion of OL differentiation in the brain in the early neonatal period would result in an increase in myelin and improvement in function.

\footnotetext{
${ }^{1}$ Department of Neurology, University of Wisconsin, Madison, Wisconsin; ${ }^{2}$ Department of Ophthalmology, Rheinische Friedrich Wilhelms University, Bonn, Germany; ${ }^{3}$ School of Pharmacy and Department of Chemistry, University of Wisconsin, Madison, Wisconsin; ${ }^{4}$ Department of Psychiatry, Washington University, St Louis, Missouri; ${ }^{5}$ Department of Medical Sciences, School of Veterinary Medicine, University of Wisconsin, Madison, Wisconsin. Correspondence: Chrysanthy Ikonomidou (Ikonomidou@neurology.wisc.edu) 


\section{Clemastine in a myelination disorder Articles}

In our study, we used two rat models for PMD, the myelindeficient (md) rat and the $p l p$ transgenic ( $p l p$-tg) rat. The $\mathrm{md}$ rat has a point mutation in the third exon of the PLP1 gene $(14,15)$, develops tremors at approximately 10 days of age, seizures at 18-20 days, and dies at 24-25 days. There is practically no myelin throughout the CNS, with a reduction in mature OLs through their early apoptosis (16-18). The scarce myelin sheaths present in the md rat lack the normal double leaflet intra-period line.

The plp-tg rat overexpresses PLP1 and was originally produced by microinjection of murine genomic Plp1 sequences into the fertilized eggs of Lewis rats. The murine PLP1 gene is $98.7 \%$ homologous to the rat gene, but shares complete protein sequence identity. The plp-tg rat models a severe form of connatal PMD $(10,19)$. The homozygous $p l p-\mathrm{tg}$ rat develops whole-body tremors and tonic-clonic seizures at postnatal days $12-14$ and dies by postnatal days $21-23$. This clinical course is equivalent to the connatal form of PMD and is almost identical to that of the md rat.

This study was designed to test the potential of clemastine fumarate to improve neurologic disease phenotype, survival, and promote CNS myelination in $m d$ and homozygous $p l p$ transgenic rats.

\section{METHODS}

All animals were maintained and utilized according to institutional standards and guidelines of the National Institutes of Health. The experimental protocol was approved by the Institutional Animal Care and Use Committee (IACUC) of the University of Wisconsin Madison.

We subjected $p l p$-tg and male md rat pups to daily subcutaneous (s.c.) injections of clemastine fumarate at three doses, 10, 20, and $30 \mathrm{mg} / \mathrm{kg} /$ day or normal saline $(10 \mathrm{ml} / \mathrm{kg} /$ day $)$ for a period of 3 weeks, starting on postnatal day 1 . Clemastine fumarate was obtained from TOCRIS Biosciences (Minneapolis, MN) and was dissolved in water.

Phenotypic analyses were performed by the same investigator blinded to genotype and therapy group. Motor phenotypes were assessed daily and body weight was continuously recorded.

\section{Genotyping}

Genotyping was performed from tail biopsies taken at postnatal days 5-10. Genomic DNA isolated from tail biopsies was used as the template in PCR to distinguish wild-type rats from hemizygous and homozygous $p l p$ rats and carriers from non-carriers md rats. $p l p$-tg-F (5'-CAG GTG TTG AGT CTG ATC ACA CAA G-3') and PLP-tg-R (5'-GCA TAA TAC GAC TCA CTA TAG GGA TC-3') primers and $m d$ 3-1: $5^{\prime}$-TTC ATG CTT TCC AGT ATG TCA TCT-3' and MD 32: 5'-GGA TGT CCT AGC CAT TTT CCC AAA-3' primers were used with Taq polymerase (Promega, Madison, WI) and the following cycling conditions for plp rats: $94^{\circ} \mathrm{C}$ for 5 min; 35 cycles of $60 \mathrm{~s}$ each at: 94,60 , and $72^{\circ} \mathrm{C}$ for $60 \mathrm{~s}$, followed by a 7 -min incubation at $72{ }^{\circ} \mathrm{C}$. Bands were visible for hemizygous and homozygous animals upon electrophoresis of PCR reactions but not for wild-type animals, as these primers recognized sequences specific to the PLP1 transgene. Identification of homozygous vs. hemizygous animals was then done based on neurologic phenotype. Hemizygous animals are normal, whereas homozygous develop tremor, ataxia, seizures, and die in the fourth week of life.

For md rats, the following cycling conditions were applied: $94{ }^{\circ} \mathrm{C}$ for $5 \mathrm{~min}, 30$ cycles at $94{ }^{\circ} \mathrm{C}$ for $30 \mathrm{~s}, 50^{\circ} \mathrm{C}$ for $30 \mathrm{~s}, 72^{\circ} \mathrm{C}$ for $30 \mathrm{~s}$ followed by $72{ }^{\circ} \mathrm{C}$ for $7 \mathrm{~min}$. PCR products were subjected to restriction digest with AvaII restriction enzyme. Expected band sizes are as follows: $\mathrm{WT}=248 \mathrm{bp}$; $m d$ carrier $248 \mathrm{bp}$ and $215 \mathrm{bp}$; affected males: $215 \mathrm{bp}$.

\section{Tissue Processing}

At age 21 days, animals were killed with an overdose of sevoflurane and were transcardially perfused with cooled $0.01 \mathrm{M}$ PBS, pH 7.4 followed by perfusion with $4 \%$ paraformaldehyde in phosphatebuffered saline. Brains, optic nerves, and spinal cords were postfixed for 3-4 days in $4 \%$ paraformaldehyde at $4{ }^{\circ} \mathrm{C}$. One hemisphere was embedded in paraffin, sagittal sections of $10 \mu \mathrm{m}$ thickness were prepared at $0.2,0.4$, and $0.6 \mu \mathrm{m}$ from midline and stained with Luxol fast blue (LFB, Sigma, Milwaukee, WI) or anti-adenomatous polyposis coli (APC) antibody (Santa Cruz, Dallas, TX), a marker for mature oligodendrocytes. The other hemisphere was used to obtain $70 \mu \mathrm{m}$ thick sagittal vibratome sections at $0.2,0.4$, and $0.6 \mu \mathrm{m}$ from midline that were processed for myelin basic protein (MBP) immunohistochemistry.

For immunostaining with MBP (1:100; Thermo Fisher Scientific, Hanover Park, IL) or APC (1:650, Santa Cruz, Dallas, TX), we used the Vectastain Elite ABC kit with Vector VIP as chromogen (Vector laboratories, Burlingame, CA). For LFB staining, sections were deparaffinized, rehydrated, and stained overnight in LFB solution at $56{ }^{\circ} \mathrm{C}$.

Terminal deoxynucleotidyl transferase (TdT) dUTP Nick-End Labeling (TUNEL) staining was used to identify apoptotic nuclei. For TUNEL staining (TACS TdT DAB in situ apoptosis detection kit, Trevigen, Gaithersburg), sections were deparaffinized and processed according to the manufacturer's instructions.

Spinal cords were sectioned in 1-mm-thick blocks, postfixed with $2.5 \%$ glutaraldehyde, osmicated, dehydrated, and embedded in Epon plastic. 1-2-mm-long sections of the optic nerves were processed for plastic embedding in the same manner. Semithin sections $(1 \mu \mathrm{m})$ were cut on a microtome and stained for myelin with $1 \%$ toluidine blue/ $1 \%$ sodiumborate.

\section{Stereology}

The numerical densities of MBP immunopositive fiber bundles (Figure $\mathbf{4 b}$ ) were estimated in the ventral brainstem (trapezoid body, longitudinal fasciculus pons, medial lemniscus, trigeminothalamic tract, pyramidal tract; areas identified using the atlas of Paxinos and Watson (20). A fiber bundle is defined as a conglomerate of longitudinally directed MBP immunopositive fibers. To be considered for counting, a fiber bundle needed to have a length of $\geq 18 \mu \mathrm{m}$ (the length of one quadrant of the counting frame at the selected magnification). The numerical densities of MBP immunopositive bundles were estimated using the stereological dissector method (21). Counts were performed using a microscope (Zeiss Axioplan 2) equipped with a $\times 10$ objective and a counting frame with dimensions $0.18 \mathrm{~mm} \times 0.18 \mathrm{~mm}$, dissector height $0.07 \mathrm{~mm}$. Eight dissectors were applied. The mean numerical densities of MBPpositive bundles $/ \mathrm{mm}^{3}\left(N_{\mathrm{v}}\right)$ were calculated.

Quantification of numerical densities of APC immunopositive oligodendrocytes (Figure 4d) was performed in the pontine nuclei using a $\times 10$ objective and a counting frame with dimensions $0.45 \mathrm{~mm} \times 0.45 \mathrm{~mm}$, dissector height $0.01 \mathrm{~mm}$. Quantification of MBP immunopositive myelin islands (Figure 4f) was performed in the hippocampus using a 6.5 objective and a counting frame with dimensions $0.71 \mathrm{~mm} \times 0.71 \mathrm{~mm}$, dissector height $0.07 \mathrm{~mm}$.

Quantification of TUNEL-positive cells (Figure 4e) was performed in the corpus callosum and the cerebellar white matter using a $\times 10$ objective and a counting frame with dimensions $0.45 \mathrm{~mm} \times 0.45 \mathrm{~mm}$, dissector height $0.01 \mathrm{~mm}$.

\section{Statistical Analysis}

Values are presented as means \pm SEM. Comparisons among groups were made using one-way ANOVA with Tukey's post hoc test or unpaired Student's $t$-test, as appropriate. The number of animals investigated in each group is stated in the figures. 


\section{Articles | Turski et al.}

\section{Mass Spectrometry}

To assess whether clemastine penetrates the blood brain barrier, we injected 6 wild-type 21-day-old Wistar rats with clemastine, $30 \mathrm{mg} / \mathrm{kg}$ s.c. and killed the animals at $6 \mathrm{~h}$ after the injection. Animals were perfused with $\mathrm{PBS}$ and their brains removed. One entire hemisphere was frozen on dry ice and processed for detection of clemastine.

\section{Sample Preparation}

Liquid-liquid extraction was performed to extract clemastine from brain tissue as reported by Xie et al. (22) with modifications. Briefly, rat brain tissue was manually homogenized in a $10-\mathrm{ml}$ glass tissue grinder with $4.3 \mathrm{ml}$ extraction solution (70\% ethyl acetate, $29 \% \mathrm{H}_{2} \mathrm{O}$, $1 \%$ methanol with $46.5 \mathrm{mM} \mathrm{NaOH}$ ). The homogenized sample was gently vortexed for $15 \mathrm{~min}$, centrifuged at 3,000 rpm for $10 \mathrm{~min}$ and the organic layer was transferred to a new tube. The liquid-liquid extraction procedure was repeated three times by replenishing $1 \mathrm{ml}$ ethyl acetate each time. The organic layers were combined and evaporated to dryness in a SpeedVac. The dried sample was reconstituted with $50 \mu \mathrm{l}$ methanol.

A MALDI-LTQ-Orbitrap XL mass spectrometer (Thermo Fisher Scientific, Bremen, Germany) was used for mass spectrometry analysis. Alpha-cyano-4-hydroxycinnamic acid matrix solution $\left(10 \mathrm{mg} / \mathrm{ml}\right.$ in $84 \%$ acetonitrile, $13 \%$ ethanol, $3 \% \mathrm{H}_{2} \mathrm{O}$ and $0.003 \%$ trifluoroacetic acid) was premixed with the sample solution at $1: 1$ (v:v) ratio. One microliter of the matrix/sample mixture was spotted onto the MALDI plate. A $\mathrm{m} / \mathrm{z}$ range of $100-600$ with resolution of 60,000 (at $\mathrm{m} / z 400)$ and laser energy of $6 \mu \mathrm{J}$ was used for all measurements. Xcalibur (Thermo Fisher Scientific, Bremen, Germany) was used for data analysis
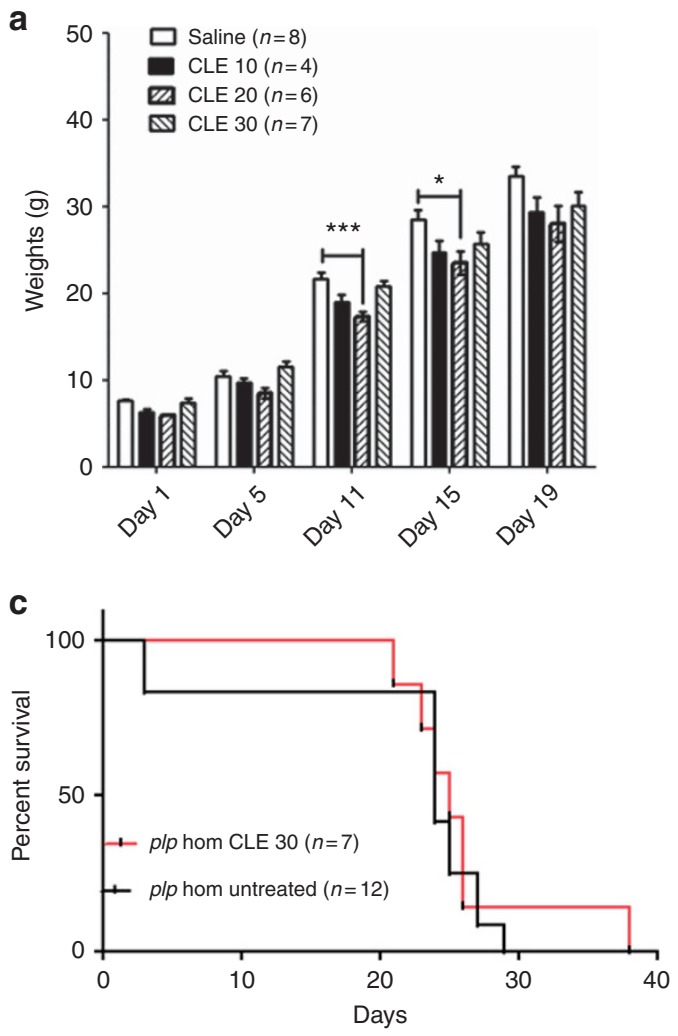

\section{RESULTS}

Daily clemastine or saline injections were tolerated well. The higher daily doses of clemastine, 20 and $30 \mathrm{mg} / \mathrm{kg}$, induced mild sedation lasting 1-2 h. In plp-tg rats, weight gain lagged compared to healthy littermates but this was not the case with md rats (Figure 1a, b). In fact, md rats treated with higher doses of clemastine thrived better in the third week of life compared to those treated with saline or lower doses of clemastine.

Both $m d$ and homozygous plp-tg rats developed the classical neurologic phenotypes. At 12-14 days of age, they developed whole-body tremor and progressive ataxia that worsened in the third week of life, which then resulted in the loss of righting reflex and death by age 24-30 days. Survival analysis comparing survival in untreated and clemastine-treated $p l p-t g$ homozygous (hom) rats and $m d$ male rats revealed no beneficial effect of clemastine $(30 \mathrm{mg} / \mathrm{kg} /$ day $)$ on overall survival (Figure 1c, d).

Histological analysis of LFB stained sections of 21-day-old $p l p$ homozygous and $m d$ male rats revealed highly abnormal myelination patterns in the entire forebrain as opposed to robust myelination in control rats (Figure 2). MBP immunostaining revealed densely myelinated fibers in control rats (Figure 3a, d) and highly fragmented or absent myelin fibers in $p l p$ homozygous and $m d$ males rats (Figure $3 \mathbf{b}, \mathbf{c})$. In many areas of the brain we saw "myelin islands",
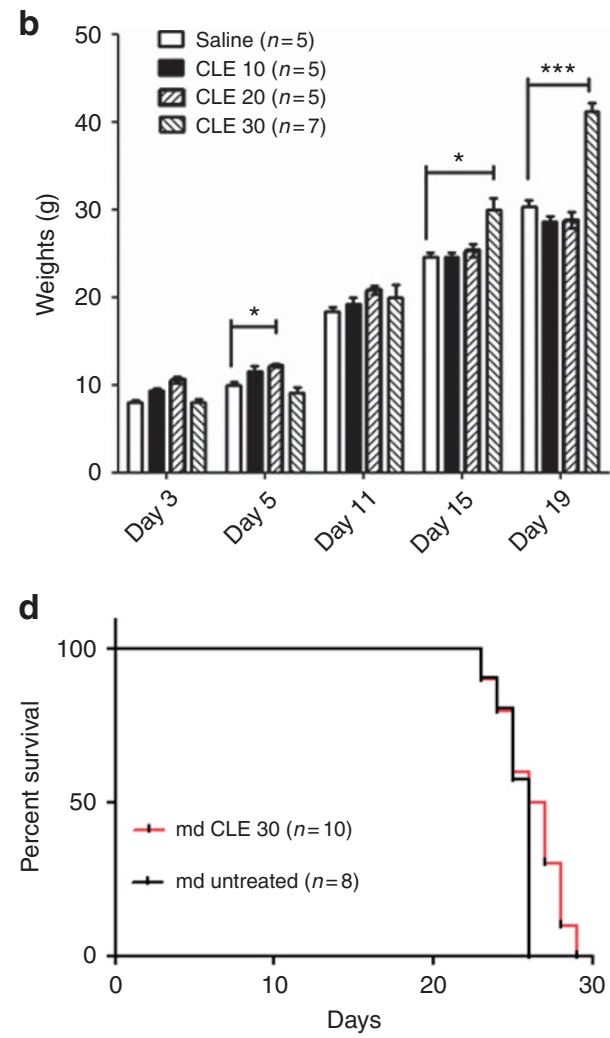

Figure 1. Weights and survival analysis in plp homozygous ( $p / p$ hom) and md male rats following treatment with saline or clemastine (CLE) (daily dose $10-30 \mathrm{mg} / \mathrm{kg}$ ) for a total of 21 days. Clemastine slowed down weight gain in plp hom (a) but not md male rats (b). In fact, md rats seemed to thrive better on clemastine $30 \mathrm{mg} / \mathrm{kg}$ during their third week of life. Survival analysis (c, d) revealed no difference in survival between plp hom untreated rats and those treated with clemastine $30 \mathrm{mg} / \mathrm{kg} /$ day for 21 days (CLE 30). Similarly, no difference in survival was achieved by treatment of $\mathrm{md}$ male rats with clemastine $30 \mathrm{mg} / \mathrm{kg} /$ day over 21 days as opposed to untreated md male rats (log-rank (Mantel-Cox) test). 


\section{Clemastine in a myelination disorder 1 Articles}
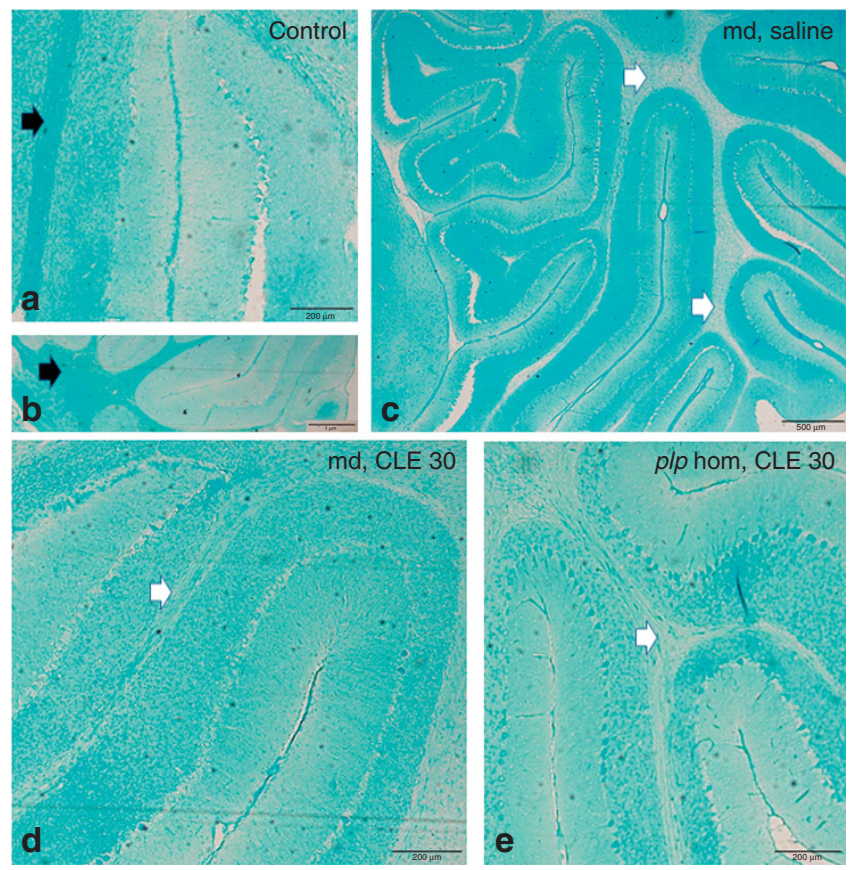

Figure 2. Lack of myelination in the brains of md male and homozygous $p / p$ rats ( $p / p$ hom). Luxol fast blue staining demonstrates robust myelination (dense green staining) in the cerebellar white matter in control rats (a, b, dark arrows). There is lack of myelin (white arrow) in the cerebellar white matter in a male md saline (c) and clemastine (daily dose of $30 \mathrm{mg} / \mathrm{kg}$ over 21 days, CLE 30) treated rat (d) and a homozygous plp clemastine-treated rat (daily dose of $30 \mathrm{mg} / \mathrm{kg}$ over 21 days). Bars $=200 \mu \mathrm{m}$ (a); $1 \mathrm{~mm}$ (b); $500 \mu \mathrm{m}$ (c); $200 \mu \mathrm{m}$ (d, e). CLE, clemastine. which represent small bundles of multidirectional MBP immunopositive fibers surrounded by large areas of absent myelin (Figure $3 \mathbf{b}$ ). Plastic sections of the optic nerves and spinal cord (Figure 3f) revealed absence of myelinated axons in both plp homozygous and $m d$ males rats.

Histological analysis of the brains, optic nerves, and spinal cords using LFB staining, MBP immunohistochemistry, and staining of ultrathin plastic-embedded sections revealed no obvious effect of clemastine treatment on myelination patterns. We performed stereologic quantification of MBP immunopositive fibers in the brainstem, MBP immunopositive myelin islands in the hippocampus, and APC immunopositive mature oligodendrocytes in the pontine nuclei in an attempt to detect subtle effects of clemastine on components of myelination in plp homozygous and md male rats. As Figure 4 demonstrates, no significant effects of treatment with clemastine were noted with the exception of a small but significant effect of $20 \mathrm{mg} / \mathrm{kg} / \mathrm{day}$ clemastine (CLE 20) on the numerical densities of MBP immunopositive fiber bundles in the brainstem. This effect was only observed in $m d$ mutant males and not in the plp homozygous rats (Figure 4a).

To determine whether clemastine affects apoptotic death of oligodendrocytes in plp homozygous and md males rats, we quantified TUNEL-positive nuclei in the corpus callosum and cerebellar white matter. As shown in Figure $4 \mathrm{~g}$, $\mathrm{h}$, there was no significant difference of numerical densities of TUNELpositive nuclei between clemastine- and saline-treated rats of either strain in the corpus callosum. The same result was
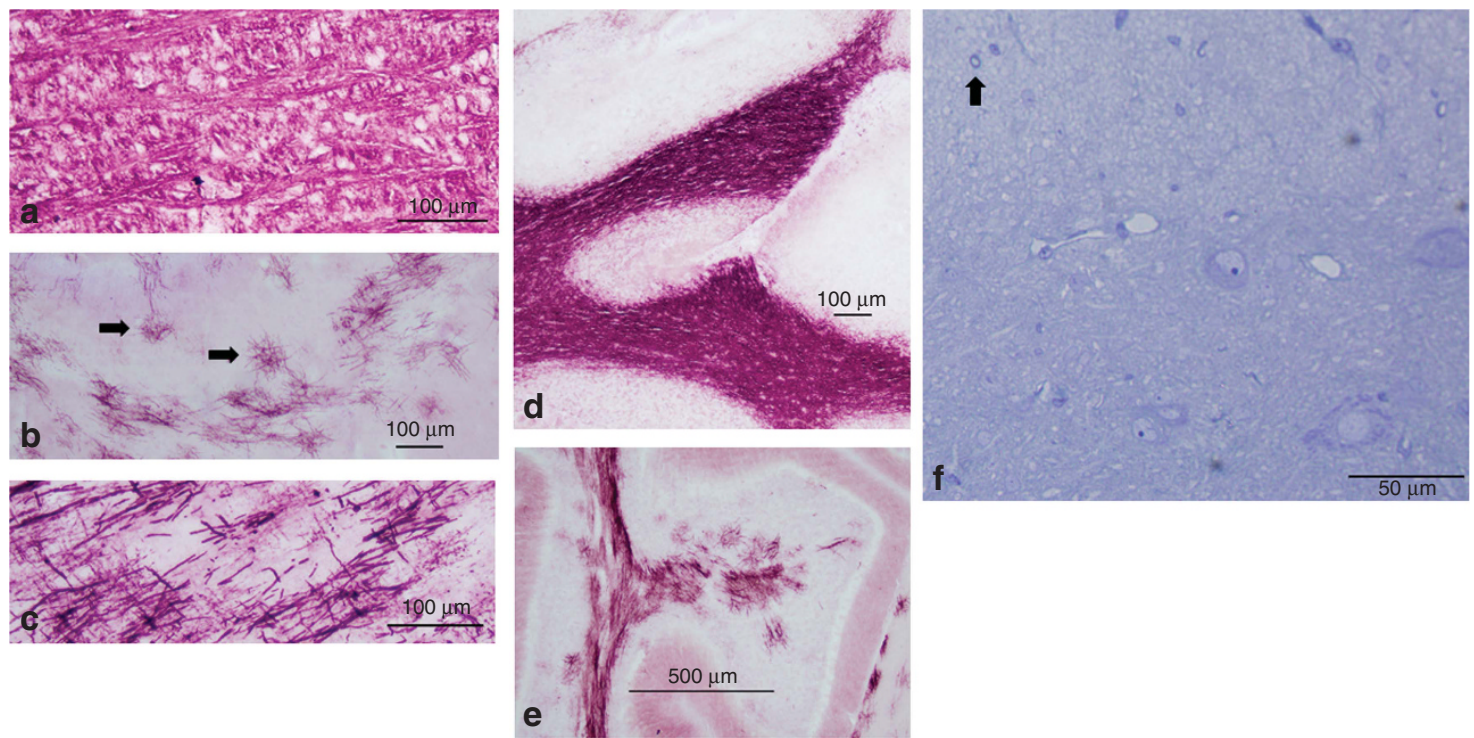

Figure 3. MBP immunohistochemistry reveals fragmented myelin and myelin islands in the brains of $p / p$ hom and md male rats. Dense immunostaining for MBP is shown in the brainstem (a) and the cerebellar white matter (d) of a 21-day-old control rat. In $\mathbf{b}$, conglomerates of multidirectional fragmented myelin fibers (myelin islands, arrows) are shown in the brainstem of a plp hom rat treated with clemastine, daily dose $30 \mathrm{mg} / \mathrm{kg}$ over 21 days. In c, another example of fragmented MBP immunopositive fibers within the pyramidal tract is shown in the same rat as in $\mathbf{b}$. (e) A light micrograph from the cerebellum of a male md rat treated with saline for 21 days. (f) A plastic section stained with toluidine blue from the spinal cord of a male md rat treated with clemastine, daily dose $30 \mathrm{mg} / \mathrm{kg}$ for 21 days. A single myelinated fiber is identified (arrow). Bars $=100 \mu \mathrm{m}$ (a-d); $500 \mu \mathrm{m}(\mathbf{e}) ; 50 \mu \mathrm{m}(\mathbf{f})$. 


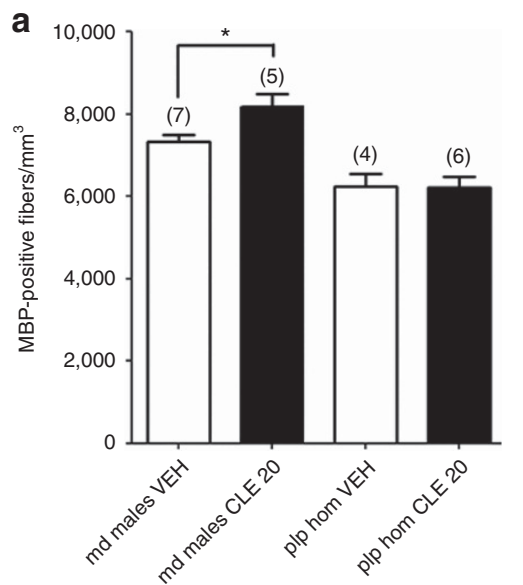

C
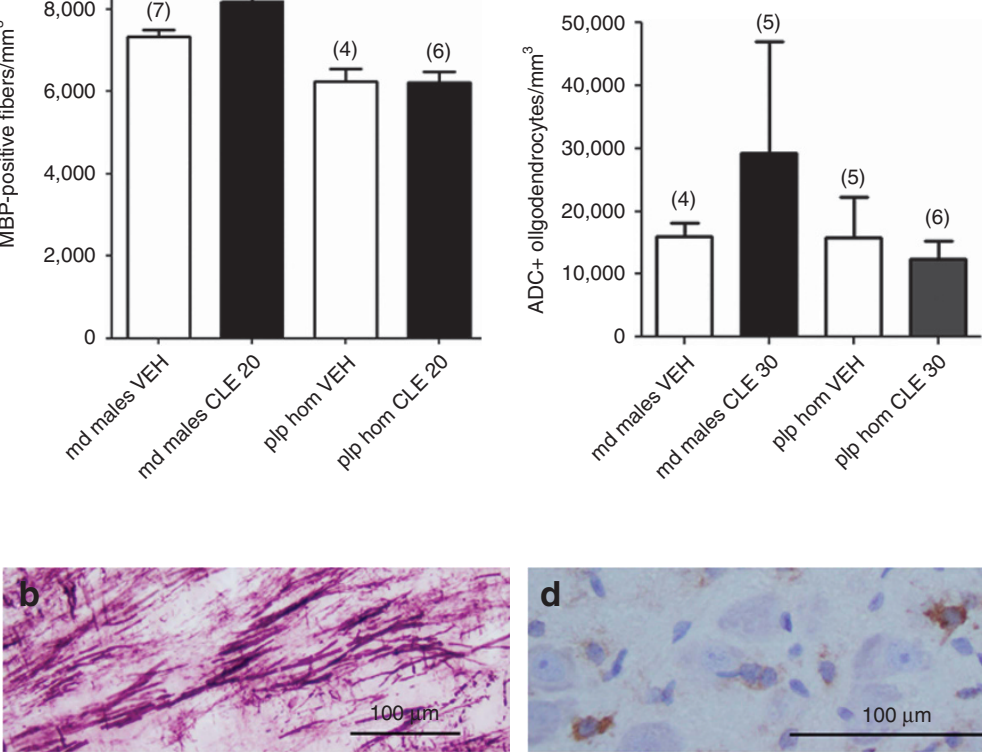

e
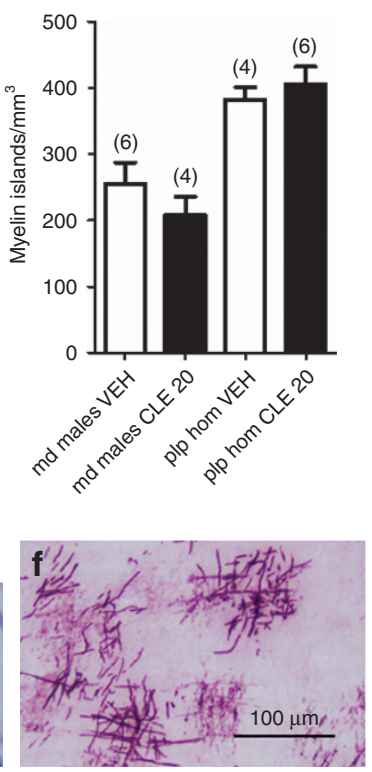

g
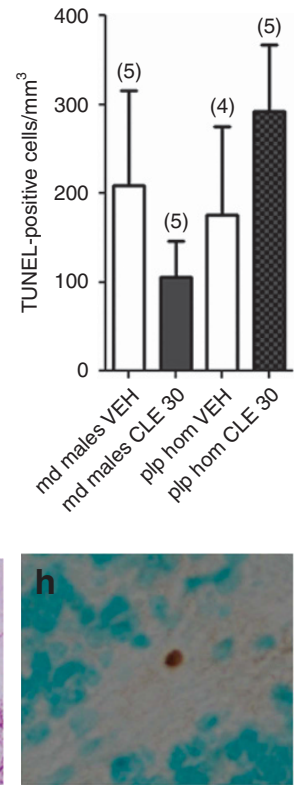

Figure 4. Stereologic quantification of numerical densities of MBP-positive fiber bundles in the brainstem (a, b), ADC+ mature oligodendrocytes (c, d) in the pons, myelin islands in the hippocampus $(\mathbf{e}, \mathbf{f})$, and TUNEL-positive cells in the corpus callosum $(\mathbf{g}, \mathbf{h})$ in the brains of md male rats or plp hom rats treated with saline (white bars) or clemastine (daily doses $20 \mathrm{mg} / \mathrm{kg}$, CLE 20, or $30 \mathrm{mg} / \mathrm{kg}$, CLE 30; black bars) for 21 days prior to euthanasia. Overall, treatment with clemastine did not significantly alter numerical densities of the evaluated histological elements with the exception of a slight increase in the numerical densities of MBP-positive fibers in CLE 20 md male rats. The histological micrographs in the bottom $(\mathbf{b}, \mathbf{d}, \mathbf{f}, \mathbf{h})$ depict the elements that were counted to produce each graph. Columns in $\mathbf{a}, \mathbf{c}, \mathbf{e}, \mathbf{g}$ and $\mathbf{h}$ represent means \pm SEM from measurements taken in $n$ animals within each group. The numbers in parentheses in the graphs indicate the number of animals in each group. Statistical comparisons between groups were performed by means of Student's $t$-test. CLE, clemastine; MBP, myelin basic protein.

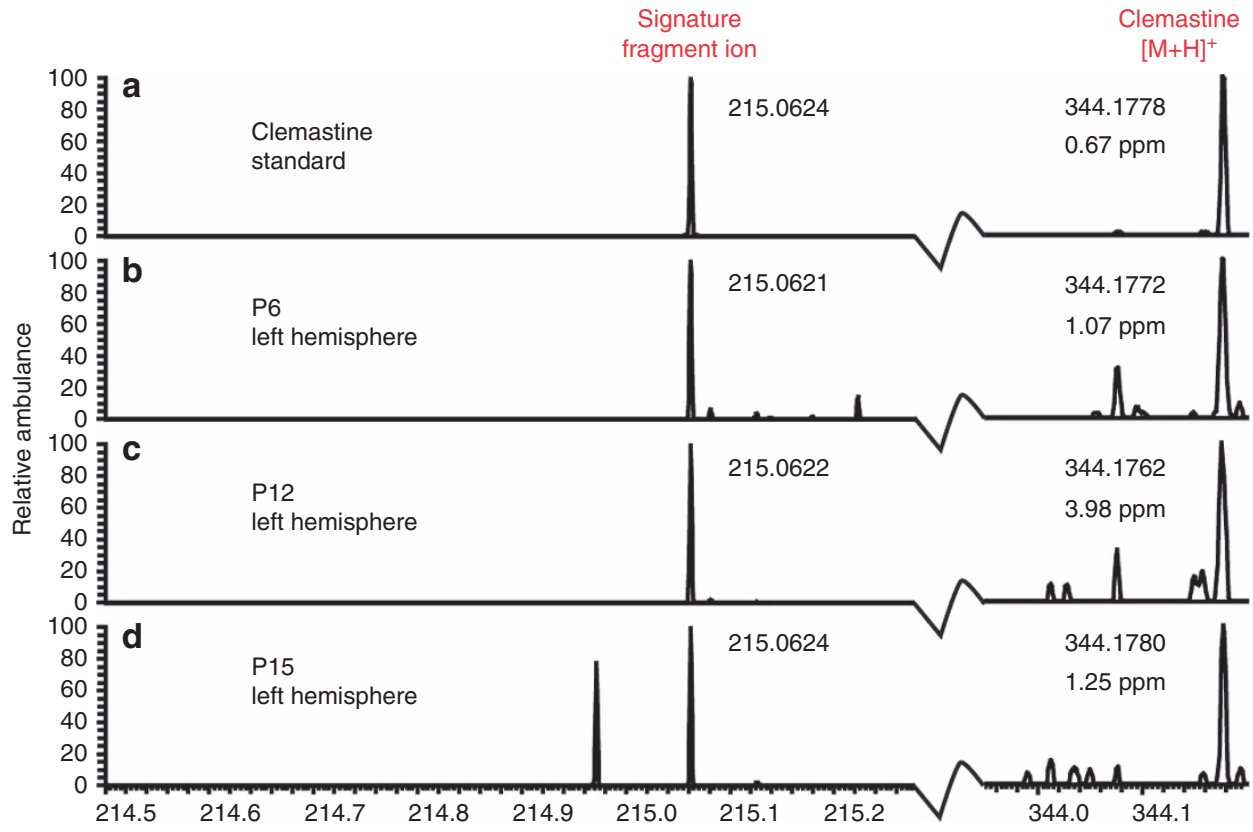

Figure 5. Clemastine is detected in brain tissue following systemic injection: MALDI-MS spectra of clemastine standard (a) and tissue homogenates from the left hemisphere (b-d) from 3 rat brains (P6, P12, P15) who had been injected $6 \mathrm{~h}$ before killing with clemastine $30 \mathrm{mg} / \mathrm{kg}$. Both the protonated parent ion at $\mathrm{m} / \mathrm{z} 344$ and a signature fragment ion at $\mathrm{m} / \mathrm{z} 215$ were observed on the spectra. 
obtained when quantifying TUNEL-positive nuclei in the cerebellar white matter.

To determine whether clemastine penetrated the blood brain barrier, we performed analysis using mass spectrometry in rat brains $6 \mathrm{~h}$ following systemic administration of $30 \mathrm{mg} /$ $\mathrm{kg}$ clemastine. As Figure 5 demonstrates, clemastine was detected in brain tissue after systemic administration.

\section{DISCUSSION}

Here we report that daily clemastine injections over 3 weeks were well tolerated in homozygous plp transgenic and male $m d$-deficient rats. Clemastine in the dose range of $10-30 \mathrm{mg} /$ $\mathrm{kg} /$ day, administered between postnatal days 1 and 21, failed to improve neurologic phenotypes, prolong survival, or improve CNS myelination as determined histologically by quantification of myelin fibers, mature oligodendrocytes, and myelin islands in selected brain regions. Clemastine did not affect apoptotic cell death in the brain. Mass spectroscopy revealed that clemastine was detectable in brain tissue indicating that it penetrated the blood brain barrier.

Our study was prompted by observations by Mayer and colleagues (12) in the shaking pup (shp), a canine model of the classical form of PMD. This study described the natural evolution of changes in the CNS of the shp. The mutation in the PLP1 in the shp leads to a delay in differentiation, increased cell death, and distension of the rough endoplasmic reticulum in oligodendrocytes. With increasing age, oligodendrocytes differentiate and survive in the spinal cord leading to myelination recovery which correlates with clinical improvement of the neurologic phenotype. These data suggest that oligodendrocytes in PMD may be more functional than previously realized and that their early recruitment could have therapeutic value. One could hypothesize that chronic pharmacotherapy with compounds that promote differentiation of OPCs would accelerate myelination in the CNS of individuals with PMD.

Clemastine is a leading candidate for myelin formation, identified from a high-throughput screening using a library containing Food and Drug Administration-approved small compounds (13). It has been shown to promote oligodendrocyte progenitor cell differentiation in vitro and remyelination after demyelinating lesions in mice $(13,23,24)$. A recent study by Li et al. (24) has shown a beneficial effect in restoring spatial working memory in mice treated with clemastine, following 6 weeks of cuprizone diet that induces demyelination. It was also recently shown to promote myelination in the prefrontal cortex and rescue behavioral phenotype in socially isolated mice (25). Although the underlying mechanism remains unclear. Subtypes of muscarinic receptors are expressed in OPCs and mature oligodendrocytes (26). Clemastine might directly act on these receptors in OPCs by favoring chromatin compaction, which plays a critical role in OPC lineage progression $(25,27)$. Clemastine promotes OPC differentiation (13) and activates histone H3K9 methyltransferase in cultured primary oligodendrocytes in the absence of neuronal or astrocytic signals.
In the study by Liu et al. (25), levels of $\mathrm{H} 3 \mathrm{~K} 9 \mathrm{me} 3$, indicative of activated histone methyltransferases, were enhanced in oligodendrocytes, but not neurons, in the PFC of socially isolated mice, thereby supporting a direct and cellautonomous effect on oligodendrocyte differentiation. An alternative mechanism of action of clemastine, as a muscarinic receptor antagonist, was suggested by the activation of Akt/mammalian target of rapamycin (mTOR) pathways induced in synaptoneurosomes by scopolamine, a nonselective muscarinic antagonist $(28,29)$. The Akt/mTOR pathway is a positive regulator of oligodendrocyte differentiation and myelination $(30,31)$. Similar signaling pathways could be activated in oligodendrocytes and result in OPC differentiation and myelination.

Despite encouraging data in the literature, we were unable to detect a beneficial effect of clemastine on neurologic and histopathological phenotypes in homozygous plp-tg and male md rats, who develop a disease phenotype that resembles connatal PMD. The doses we used are similar to those used by other investigators. Liu et al. (25) administered $10 \mathrm{mg} / \mathrm{kg}$ of clemastine daily by gastric tube over a period of 2 weeks. Furthermore, we confirmed that clemastine entered brain tissue using mass spectroscopy (Figure 5).

There are several possibilities to explain the lack of clemastine effect in our two rat models for PMD. First, the animal models we used demonstrate a very severe phenotype with death occurring in the fourth week of life. The life span of these animals might be too short to be able to detect an effect of clemastine on a very severely impaired myelination process. One might still be able to detect such a positive effect if treatment were started prenatally. Experiments with PMD animal models with longer life span, specifically the shaking pup, would be crucial to further explore the fundamental question whether clemastine and other antimuscarinic compounds are agents with therapeutic potential for PMD.

It is also possible that the plp transgene and the md mutation preclude a therapeutic effect of clemastine on affected OPCs, i.e., that OPCs in PMD do not respond to clemastine as normal OPCs do. The animal models in which clemastine has been tested so far do not have a primary myelination disorder, myelination deficits are induced by toxins, inflammation, or social deprivation. Our data do not support the hypothesis that a therapeutic effect is not seen with clemastine due to acceleration of apoptosis of OPCs.

In summary, we report here lack of therapeutic effect of chronic clemastine injections in two rat models of PMD. Our results suggest that clemastine may not be a promising agent for treating connatal forms of PMD in humans. More research is needed in animal models with milder forms of the disease and longer life span as well as human OPCs to fully explore the potential of clemastine and other antimuscarinic agents in the treatment of humans with PMD.

\section{ACKNOWLEDGMENTS}

This project was supported by research funds from the Department of Neurology, University of Wisconsin, Madison 


\section{Articles | Turski et al.}

\section{STATEMENT OF FINANCIAL SUPPORT}

This project was supported by research funds from the Department of Neurology, University of Wisconsin, Madison

Disclosure: The authors declare no conflict of interest.

\section{REFERENCES}

1. Inoue K. PLP1-related inherited dysmyelinating disorders: PelizaeusMerzbacher disease and spastic paraplegia type 2. Neurogenetics 2005;6: $1-16$.

2. Yool DA, Edgar JM, Montague P, Malcolm S. The proteolipid protein genes and myelin disorders in man and animal models. Hum Mol Genetics 2000;9:987-92.

3. Nave KA, Boespflug-Tanguy O. X-linked developmental defects of myelination: from mouse mutants to human genetic disorders. Neuroscientist 1996;2:33-43.

4. Nave KA, Millner RJ. Proteolipid proteins: structure and genetic expression in normal and myelin-deficient mutant mice. Crit Rev Neurobiol 1989;5:65-91.

5. Nave K-A, Lai C, Bloom FE, Milner RJ. Jimpy mutant mouse: a 74-base deletion in the mRNA for myelin proteolipid protein and evidence for a primary defect in RNA splicing. Proc Natl Acad Sci USA 1986;83:9264-8.

6. Nave K-A, Lai C, Bloom FE, Milner RJ. Splice site selection in the proteolipid protein (PLP) gene transcript and primary structu re of the DM-20 protein of central nervous system myelin. Proc Natl Acad Sci USA 1987;84:5665-9.

7. Werner H, Jung M, Klugmann M, Sereda M, Griffiths IR, Nave KA. Mouse models of myelin diseases. Brain Pathol 1998;8:771-93.

8. Duncan ID, Hammang JP, Trapp BD. Abnormal compact myelin in the myelin-deficient rat: absence of proteolipid protein correlates with a defect in the intraperiod line. Proc Natl Acad Sci USA 1987;84:6287-91.

9. Duncan ID, Kondo Y, Zhang S-C. The myelin mutants as models to study myelin repair in the leukodystrophies. Neurotherapeutics 2012;8:607-24.

10. Duncan ID, Radcliff AB. Inherited and acquired disorders of myelin: The underlying myelin pathology. Exp Neurol 2016;283 (Pt B): 452-75.

11. Griffiths IR, Duncan ID, McCulloch M, Harvey JA. Shaking pups: a disorder of central myelination in the Spaniel dog. I. Clinical, genetic and light microscopical observations. J Neurol Sci 1981;50:423-33.

12. Mayer JA, Griffiths IR, Goldman JE, et al. Modeling the natural history of Pelizaeus-Merzbacher disease. Neurobiol Dis 2015;75:115-30.

13. Mei F, Fancy SPJ, Shen Y-AA, et al. Micropillar arrays as a highthroughput screening platform for therapeutics in multiple sclerosis. Nat Med 2014;20:954-60.

14. Boison D, Stoffel W. Myelin-deficient rat: a point mutation in exon III (A-C, Thr-Pro) of the myelin proteolipid protein causes dysmyelination and oligodendrocyte death. EMBO J 1989;8:3295-302.
15. Simons R, Riordan JR. The myelin-deficient rat has a single base substitution in the third exon of the myelin proteolipid protein gene. J Neurochem 1990;54:1079-81.

16. Jackson KF, Duncan ID. Cell kinetics and cell death in the optic nerve of the myelin deficient rat. J Neurocytol 1988;17:657-70.

17. Grinspan JB, Coulalaglou M, Beesley JS, Carpio DF, Scherer SS. Maturation-dependent apoptotic cell death of oligodendrocytes in myelin-deficient rats. J Neurosci Res 1998;54:623-4.

18. Lipsitz D, Goetz BD, Duncan ID. Apoptotic glial cell death and kinetics in the spinal cord of the myelin-deficient rat. J Neurosci Res 1998;51: 497-507.

19. Mayer JA, Larsen EC, Kondo Y, Duncan ID. Characterization of a PLPoverexpressing rat, a model for the connatal form of PelizaeusMerzbacher disease. Neurobiol Dis 2011;44:231-8.

20. Paxinos G, Watson C. The Rat Brain in Stereotaxic Coordinates. Amsterdam: Elsevier, 2007.

21. West MJ, Gundersen HJ. Unbiased stereological estimation of the number of neurons in the human hippocampus. J Comp Neurol 1990;296:1-22.

22. Xie Z, Liao Q, Li Z, Zhu C, Zeng Y, Liu S. Development and full validation of a sensitive quantitative assay for the determination of clemastine in human plasma by liquid chromatography-tandem mass spectrometry. J Pharm Biomed Anal 2007;44:924-30.

23. Deshmukh VA, Tardif V, Lyssiotis CA, et al. A regenerative approach to the treatment of multiple sclerosis. Nature 2013;502:327-32.

24. Li Z, He Y, Fan S, Sun B. Clemastine rescues behavioral changes and enhances remyelination in the cuprizone mouse model of demyelination. Neurosci Bull 2015;31:617-25.

25. Liu J, Dupree JL, Gacias M, et al. Clemastine enhances myelination in the prefrontal cortex and rescues behavioral changes in socially isolated mice. J Neurosci 2016;36:957-62.

26. De Angelis F, Bernardo A, Magnaghi V, Minghetti L, Tata AM. Muscarinic receptor subtypes as potential targets to modulate oligodendrocyte progenitor survival, proliferation, and differentiation. Dev Neurobiol 2012;72:713-28.

27. Liu J, Dietz K, DeLoyht JM, et al. Impaired adult myelination in the prefrontal cortex of socially isolated mice. Nat Neurosci 2012;15:1621-3.

28. Voleti B, Navarria A, Liu RJ, et al. Scopolamine rapidly increases mammalian target of rapamycin complex 1 signaling, synaptogenesis, and antidepressant behavioral responses. Biol Psychiatry 2013;74:742-9.

29. Navarria A, Wohleb ES, Voleti B, et al. Rapid antidepressant actions of scopolamine: role of medial prefrontal cortex and M1-subtype muscarinic acetylcholine receptors. Neurobiol Dis 2015;82:254-61.

30. Wood TL, Bercury KK, Cifelli SE, et al. mTOR: a link from the extracellular milieu to transcriptionalregulation of oligodendrocyte development. ASN Neuro 2013;5:e00108.

31. Wahl SE, McLane LE, Bercury KK, Macklin WB, Wood TL. Mammalian target of rapamycin promotes oligodendrocyte differentiation, initiation and extent of CNS myelination. J Neurosci 2014;34:4453-65. 